\title{
Design and Performance of a High-Repetition-Rate Single-Frequency Yb:YAG Microlaser
}

\author{
Randal L. Schmitt ${ }^{\dagger}$ and Binh T. Do \\ Sandia National Laboratories \\ Albuquerque, NM 87185
}

\begin{abstract}
We describe the design and performance of a high-repetition-rate single-frequency passively Q-switched Yb:YAG microlaser operating near $1030 \mathrm{~nm}$. By using short cavity length, an intracavity Brewster polarizer, and an etalon output coupler, we are able to produce $\sim 1$-ns-long single-frequency pulses at repetition rates up to $19 \mathrm{kHz}$ without shot-to-shot mode hopping. The laser's output spatial mode is $\mathrm{TEM}_{00}$ and its pulse energy varies between $31 \mu \mathrm{J}$ and $47 \mu \mathrm{J}$ depending on repetition rate. Its peak optical-to-optical efficiency is $22 \%$.
\end{abstract}

Keywords: microlaser; microchip laser, passively Q-switched, $\mathrm{Yb}: \mathrm{YAG}, \mathrm{Cr}^{4+}: \mathrm{YAG}$, diode-pumped, nanosecond

\section{INTRODUCTION}

Diode-laser-pumped passively Q-switched microlasers have many applications in ladar (laser ranging), lidar (remote sensing), materials processing, micro surgery, micro LIBS (laser induced breakdown spectroscopy), and others. These miniature lasers are capable of producing single-longitudinal-mode, diffraction-limited output in sub-nanosecond to nanosecond-long pulses. The high-beam-quality, high-irradiance laser light from a microlaser is ideal for efficient nonlinear optical frequency conversion to the ultraviolet or the infrared, enabling the miniaturization of spectroscopicbased instrumentation and compact lidar systems. Most recently, microlasers have been used as front-end seed sources for fiber amplifiers, and have the potential for seeding multi-pass bulk amplifiers or even disk amplifiers. We have been developing a family of $\mathrm{Yb}: \mathrm{YAG}$ microlasers with pulse lengths from $0.5 \mathrm{~ns}$ to $1.0 \mathrm{~ns}$ and single longitudinal mode operation at repetition rates up to $19.4 \mathrm{kHz}$ to use as seed sources for fiber amplifiers. The 1030-nm output of Yb:YAG more closely matches the gain peak in Yb-doped fiber than the $\sim 1060$-nm output of Nd-based lasers and results in more efficient pulsed fiber laser systems ${ }^{1}$. The microlaser described in this paper has been configured to produce $\sim 1$-ns-long pulses and has been optimized for single-mode operation at high repetition rate.

Over a decade ago, the first diode-laser-pumped passively Q-switched microlasers were demonstrated with a self-Qswitched Cr,Nd:YAG laser ${ }^{2}$ and shortly thereafter, a composite $\mathrm{Cr}^{4+}: \mathrm{YAG} / \mathrm{Nd}: \mathrm{YAG}$ laser ${ }^{3}$. Since that time, there has been a great deal of research and development in the field of diode-laser-pumped passively Q-switched microlasers, resulting in microlasers that span a variety of wavelengths, and a better understanding of how to design and predict the performance of these lasers ${ }^{4-7}$. A number of vendors now provide both custom and off-the-shelf microlaser products. Certainly the most common passively Q-switched microlasers built to date use Nd:YAG as the gain medium and $\mathrm{Cr}^{4+}$ :YAG as the saturable absorber because of the excellent optical, mechanical, and thermal properties of the YAG host as well as the ready availability of both materials in high-quality crystals (and now in ceramic form). Additionally, the gain crystal and the Q-switch can be diffusion bonded together to form a monolithic laser. More recently, Yb:YAG has been used as the gain medium in microlasers ${ }^{8-15}$.

Yb:YAG has a number of advantages over Nd:YAG as a gain medium, including lower quantum defect ${ }^{16}(11 \%$ in $\mathrm{Yb}: \mathrm{YAG}$ compared to $24 \%$ in Nd:YAG) which results in more efficient pumping and lower heat deposition, and broader absorption bandwidth ( $\sim 18 \mathrm{~nm}$ wide for Yb:YAG vs. $<4 \mathrm{~nm}$ wide for Nd:YAG) which allows greater tolerance on the pump laser wavelength. Moreover, $\mathrm{Yb}$ can be doped into $\mathrm{YAG}$ at higher concentration without suffering concentration

\footnotetext{
† corresponding author: schmitt@sandia.gov

${ }^{*}$ current affiliation: Ball Aerospace \& Technologies Corp., Albuquerque, NM
}

Solid State Lasers XVII: Technology and Devices 
quenching ${ }^{17}, 18$. In addition, the lower stimulated emission cross section of $\mathrm{Yb}$ :YAG results in higher saturation fluence and higher stored energy before bleaching the Q-switch. This results in shorter pulse length, higher pulse energy, and higher Q-switched pulse extraction efficiency compared to a similar Nd-based microlaser ${ }^{4,7,12,19}$. Also, the passively Qswitched Yb:YAG laser lacks the undesirable afterpulse that frequently plagues Nd-based microlasers. However, the emission bandwidth of $\mathrm{Yb}: \mathrm{YAG}$ is about $6 \mathrm{~nm}$ wide, approximately 10 times broader than that of $\mathrm{Nd}: \mathrm{YAG}^{16}$, presenting a challenge to build a nanosecond passively Q-switched Yb:YAG laser that operates on a single longitudinal mode without shot-to-shot mode hopping at higher repetition rates. Many applications of microlasers, particularly spectroscopic applications, require reliable single-longitudinal mode operation, and this has been a focus of our Yb:YAG microlaser development effort.

Because of its performance advantages, Yb:YAG has been the subject of active research and development, including its use in passively Q-switched microlasers. Fan et al. ${ }^{8}$ reported the first diode-laser-pumped Yb:YAG laser in 1993. It was electro-optically Q-switched and produced 11-ns long pulses with an energy of $72 \mu \mathrm{J} /$ pulse. In 2001, Spühler and coworkers $^{9}$ demonstrated the first passively Q-switched Yb:YAG microlaser using a 200 - $\mu \mathrm{m}$-thick piece of 20 -at\%-doped $\mathrm{Yb}$ :YAG and a semiconductor saturable absorber (SESAM) Q-switch. Because of its extremely short cavity length, this microlaser was able to output 530-ps-long single-longitudinal-mode pulses with $1.1 \mu \mathrm{J} /$ pulse at $12 \mathrm{kHz}$ repetition rate. Also in 2001, Dong et al. ${ }^{10}$ were the first to use $\mathrm{Cr}^{4+}$ :YAG to passively Q-switch a Yb:YAG laser. Their Ti:sapphirepumped system generated pulses as short at $350 \mathrm{~ns}$ with $3.2 \mu \mathrm{J} /$ pulse at $17 \mathrm{kHz}$. In 2002, Shen et al. ${ }^{11}$ demonstrated a passively Q-switched $\mathrm{Yb}$ :YAG laser using a thin piece of single-crystal GaAs as the saturable absorber and output coupler. Their laser produced 15.5 -ns-wide pulses with $75.6 \mu \mathrm{J} /$ pulse at $7.3 \mathrm{kHz}$ repetition rate. Ostby et al. ${ }^{13}$ conducted a parametric study with a $\mathrm{Cr}^{4+}$ :YAG saturable absorber and 10-at\%-doped $\mathrm{Yb}: \mathrm{YAG}$ to find the optimum saturable absorber density and output coupling for a $\sim 1$-ns ladar transmitter. Their most efficient design produced 1.7-ns-long pulses with $68 \mu \mathrm{J} /$ pulse at $10 \mathrm{kHz}$, although the laser mode hopped shot to shot, and, in some cases, the polarization flipped. Later, Ostby and co-workers ${ }^{20}$ operated their microlaser using ceramic Yb:YAG and demonstrated that their ceramic material was competitive with crystalline $\mathrm{Yb}: \mathrm{YAG}$ in terms of performance. In order to force operation on a single linear polarization, they put a small Brewster window inside the laser cavity. The ceramic Yb:YAG laser operated multi-longitudinal mode producing 1.9-ns-long pulses with $63 \mu \mathrm{J} /$ pulse at $11.4 \mathrm{kHz}$. Dong et $\mathrm{al}^{21}$ built an all-ceramic microlaser with 1-mm-thick 9.8-at\%-doped ceramic Yb:YAG gain section and 0.2-mm-thick ceramic $\mathrm{Cr}^{4+}$ :YAG Qswitch which produced 380-ps-long pulses with about $30 \mu \mathrm{J} /$ pulse. The laser operated single longitudinal mode at repetition rates up to $4 \mathrm{kHz}$ and the authors noted that when the laser began mode hopping, it did not operate on adjacent longitudinal modes, a fact they attributed to the etalon effect of the uncoated Q-switch.

\section{YB:YAG MICROLASER DESIGN}

A schematic of our $\mathrm{Yb}$ :YAG microlaser is shown in Figure 1. The $\sim 3-\mathrm{mm}$-long laser cavity consists of a 3-mm x 3-mm $\mathrm{x}$ 1-mm-thick 10-at.\% doped Yb:YAG gain section, a 0.3-mm-thick $\mathrm{Cr}^{4+}$ :YAG Q-switch, a miniature Brewster-angle polarizer, and a 300- $\mu \mathrm{m}$-thick undoped YAG etalon output coupler. The back surface of the Yb:YAG gain crystal has a dual coating that has high transmission at the $940-\mathrm{nm}$ pump wavelength and high reflectivity at $1030 \mathrm{~nm}$ and serves as the rear mirror of the laser cavity. The front surface of the Yb:YAG crystal has an anti-reflection coating for $1030 \mathrm{~nm}$ and a high reflectivity coating for $940 \mathrm{~nm}$ to double pass the pump light for lower threshold and increased efficiency. The $0.3-\mathrm{mm}$-thick $\mathrm{Cr}^{4+}$ :YAG Q-switch has an unsaturated transmission of $93 \%$ at $1030 \mathrm{~nm}$ and is anti-reflection coated for $1030 \mathrm{~nm}$ on both faces. In order to ensure that the microlaser operates on a single linear polarization on every shot, particularly at higher repetition rates, we designed a miniature intracavity Brewster-angle polarizer. It consists of a 150$\mu \mathrm{m}$-thick plane-parallel fused-silica window held at Brewster's angle in a 3-mm x 3-mm x 1-mm-thick aluminum frame. Finally, in order prevent shot-to-shot mode hopping at higher repetition rates, and to serve as the output coupler, we use a $300-\mu \mathrm{m}$-thick undoped YAG etalon ${ }^{11,22,23}$. At anti-resonance the uncoated YAG etalon $(\mathrm{n}=1.82)$ has about $28 \%$ reflectivity which is nearly optimal output coupling for this microlaser.

As mentioned previously, the wide gain bandwidth of Yb:YAG and spatial hole burning in the standing-wave cavity makes single-longitudinal mode operation a challenge, particularly at repetition rates $>1 \mathrm{kHz}$. Although minimizing the length of the cavity (and thereby minimizing the number of modes under the gain curve) is necessary, it is not sufficient to ensure single-mode operation in Yb:YAG at higher repetition rates. Fortunately, on any single pulse, the passively Qswitched laser operates on a single longitudinal mode due to spatial hole burning in the saturable absorber Q-switch. This 
is because the first mode to reach threshold bleaches a standing wave pattern in the saturable absorber, making it difficult for other modes to reach threshold. We purposefully place the Q-switch near the center of the cavity to maximize this effect ${ }^{24}$. Since the lifetime of the upper level in $\mathrm{Cr}^{4+}: \mathrm{YAG}$ is $\sim 3 \mu \mathrm{s}$, the standing-wave pattern in the Q-switch is erased before each subsequent shot, even at high repetition rates. Since the fluorescence lifetime in Yb:YAG is nearly $1 \mathrm{~ms}$, spatial hole burning in the gain medium can cause shot-to-shot mode hopping at repetition rates $>1 \mathrm{kHz}$. Recently, Dong et al. ${ }^{14}$ modeled the cross saturation effects of spatial hole burning for passively Q-switched microlasers and their results agree with observed microlaser behavior: at higher repetition rates where mode hopping occurs, the mode hopping is not random, but follows a regular pattern determined by the length of the gain medium, the pump power level and pump focus geometry, among other factors. In addition, the lasing modes are not, in general, adjacent longitudinal modes (due to the fact that the gain medium is thin and located near one end of the cavity).

To obtain reliable single-longitudinal mode operation at the highest possible repetition rates from our microlaser, we used an etalon output coupler. We initially experimented with an intracavity etalon but found that it was difficult to mount inside the heat sink assembly and impossible to adjust after installation. By combining the output coupler with the etalon, mounting issues are simplified and the tilt of the etalon can be adjusted to tune its peak reflectivity to coincide with a cavity mode near the peak gain.

The $\mathrm{Yb}: \mathrm{YAG}$ gain crystal, $\mathrm{Cr}^{4+}: \mathrm{YAG}$ Q-switch crystal, and miniature Brewster-angle polarizer are all mounted in a copper (or aluminum) clamping $\mathrm{v}$-groove housing which serves as a heat sink. The two crystals are wrapped in a thin $(\sim 25-$ $\mu \mathrm{m}$ thick) layer of indium foil to improve heat transfer to the clamping heat sink. An adjustable mirror mount that holds the output coupler is integrated into the microlaser housing, allowing final optical alignment of the microlaser cavity. In addition, the adjustable mount allows slight tip/tilt angle tuning of the etalon without significantly affecting the symmetry or beam quality of the output beam. The mounts holding the SMA-terminated pump fiber, coupling lens, and spacers needed to adjust the pump beam size and location are all modular to allow flexibility in adjusting the pump spot size for optimum operation and to allow easy reconfiguration of the microlaser system. A complete microlaser assembly, including pump

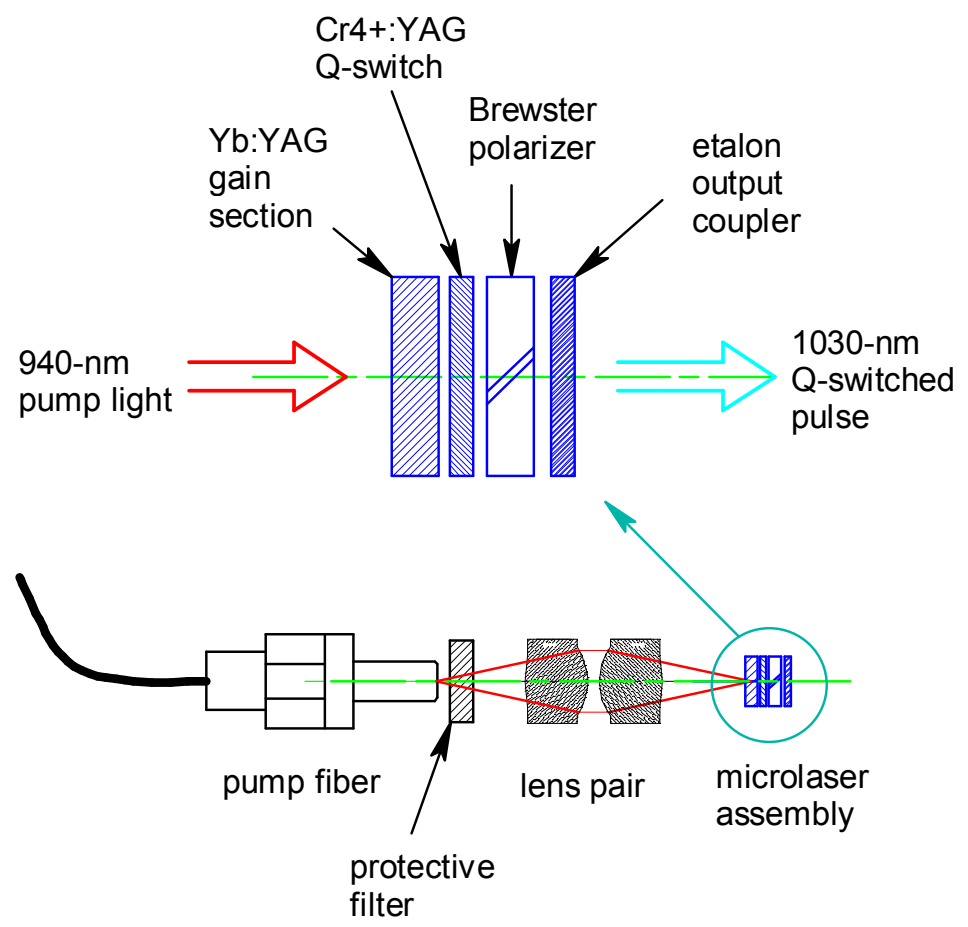

Figure 1 Typical Yb:YAG microlaser layout. The bottom illustration shows a scale drawing of the optical layout. Note the size of the SMA-905 connector on the pump fiber for scale. The details of the passively Q-switched microlaser cavity are shown directly above.

diode, is shown in Figure 2. Note that in this photo, an ordinary plane partially reflecting mirror is installed as the output coupler. The results described in this paper were obtained using a thin etalon output coupler in place of the simple partial mirror. The etalon was attached to the end face of $12.5-\mathrm{mm}$-diameter aluminum tube so that it could be held in the mirror mount and positioned as close as possible $(\sim 300 \mu \mathrm{m}$ gap) to the laser crystal holder to minimize the overall cavity length.

The microlaser assembly is mounted on a thermoelectrically cooled heat sink and its temperature is controlled to within $\pm 0.01{ }^{\circ} \mathrm{C}$ using an ILX model LDT-5901B temperature controller. We normally operated the microlaser at $10{ }^{\circ} \mathrm{C}$. We 
observed that the microlaser is more efficient and can be operated at higher repetition rates (maintaining single mode operation) at lower temperatures, but we limited the temperature to $10^{\circ} \mathrm{C}$ to avoid condensation of moisture from the air.

Our microlaser is pumped by a 7-Watt 940 -nm fiber-coupled laser diode from EM4. The core of the pump fiber is $105 \mu \mathrm{m}$ and its numerical aperture is 0.15 . The laser diode is mounted on a thermoelectrically cooled (or heated) heat sink and its temperature was kept at $45 \pm 0.03{ }^{\circ} \mathrm{C}$ (using an ILX model LDT-5901B temperature controller) to maintain a center wavelength of $\sim 938 \mathrm{~nm}$ to match the peak absorption in Yb:YAG. Although the absorption bandwidth of the 940-mn pump feature is wide, we still observe measurable changes in pulse repetition rate resulting from temperature changes in the diode laser, and, in order to maintain stable single-longitudinal-mode operation over long time periods, we found it necessary to control the laser diode temperature to $\pm 0.03{ }^{\circ} \mathrm{C}$.

The output of the pump fiber is imaged into the Yb:YAG gain crystal with $\sim 1: 1$ magnification using two highnumerical-aperture 11-mm-focal-length lenses back to back (Thorlabs C220MP) as shown in the scale drawing in Figure 1. A special dielectric optical filter is placed between the pump fiber and the lens pair to reflect any leakage of Q-switched 1030-nm light in the backward direction that could potentially damage the pump diode. This protective filter is coated to have high reflectivity at

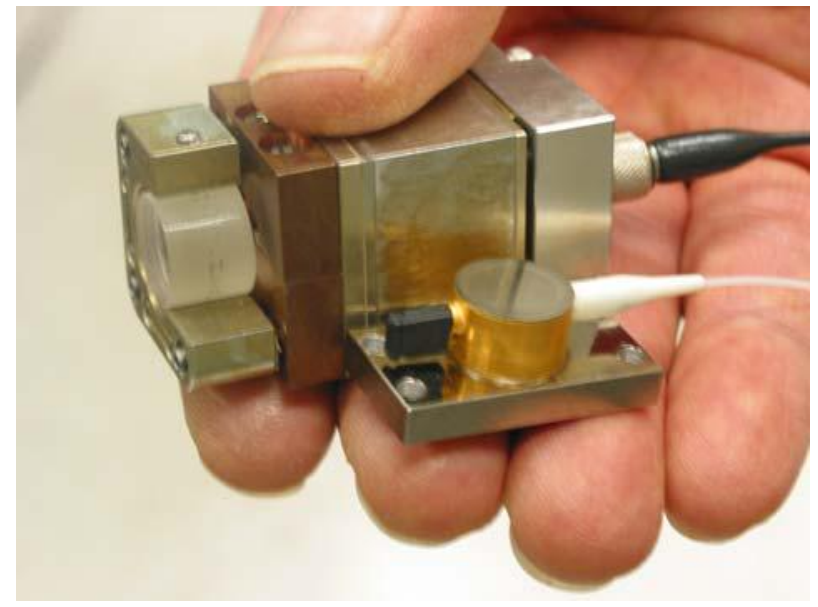

Figure 2 Photo of Yb:YAG microlaser and its pump diode. $1030 \mathrm{~nm}$ and high transmission at the $940-\mathrm{nm}$ pump wavelength.

The pump diode is driven by a Wavelength Electronics model PLD-5000 laser diode driver which can operate in any of three modes: $\mathrm{cw}$, analog modulation, or a combination of the $\mathrm{cw}$ and modulation. We operated our microlaser in both cw pump mode and also in pulsed pump mode. For pulsed pumping we set a low-level continuous current level and use an SRS DG535 digital delay generator to control the frequency (repetition rate), pulse width, and pulse amplitude of the drive current. Pulse pumping a passively Q-switched microlaser can reduce pulse-to-pulse timing jitter significantly ${ }^{25,26}$, but the technique is somewhat limited at higher repetition rates (say $>15 \mathrm{kHz}$ ) by the rise- and fall-time of the laser diode driver.

\section{YB:YAG MICROLASER PERFORMANCE}

In the following section, we discuss the performance of the Yb:YAG microlaser described above. Table 1 summarizes the output pulse energy, pulse length, and pulse-to-pulse timing jitter for the Yb:YAG microlaser operated at a number of different pulse repetition rates for both pulsed pumping and cw pumping. In all cases described in this work, the microlaser was operating single-longitudinal mode as verified by observing the fringe pattern produced by a 3-mm-thick fused silica Fabry-Perot etalon. The free spectral range of the monitor etalon was $\sim 34.5 \mathrm{GHz}$ or $1.15 \mathrm{~cm}^{-1}$. In addition, we sometimes used a grating-based mode monitor to ensure that multiple orders of the Fabry-Perot etalon did not accidentally overlap. When the microlaser did mode hop shot to shot, we also used the grating-based monitor to measure the spacing between the operating modes. We observed that the microlaser never mode hopped to the nearest longitudinal mode, but rather, always hopped three or more longitudinal modes away. This is due to the fact that the gain medium is thin and located adjacent to the rear mirror. At this position in the cavity, the standing-wave pattern burned in the gain medium is least favorable to the nearest neighbor mode hopping. (Also, the output coupler etalon precludes adjacent-longitudinal-mode hopping, but we observed this behavior even for cases when no etalon was used in the cavity.) This behavior is qualitatively consistent with the modeling results recently reported by Dong et al. ${ }^{14}$ In addition, the pulse-to-pulse timing jitter noticeably increases when the microlaser is mode hopping shot-to-shot, and we used the low timing jitter as further evidence that the microlaser was consistently operating single longitudinal mode on every shot. Excessive timing jitter is due to the fact that each longitudinal mode experiences slightly different gain, and, as a result, different Q-switched pulse build-up time. Finally, when the laser is mode hopping, the pulse-to-pulse energy 
stability is much worse than the $\sim 0.5 \%$ pulse-to-pulse stability that we typically observe when the microlaser is reliably operates on the same mode on every pulse. Again, this is due to the fact that each longitudinal mode experiences slightly different gain which results in different modes having different output energies.

Table 1 Pulse width, output energy, and timing jitter measured for different repetition rates and pump configurations.

\begin{tabular}{|c|c|c|c|c|}
\hline $\begin{array}{c}\text { rep rate } \\
(\mathbf{k H z})\end{array}$ & $\begin{array}{c}\text { pulse width } \\
\text { FWHM } \\
(\mathbf{n s})\end{array}$ & $\begin{array}{c}\text { output energy } \\
(\boldsymbol{\mu J})\end{array}$ & $\begin{array}{c}\text { pulse-to-pulse } \\
\text { timing jitter } \\
(\mathbf{n s})\end{array}$ & $\begin{array}{c}\text { pump } \\
\text { mode }\end{array}$ \\
\hline 1 & $\S$ & $30.8 \pm 0.1$ & 100 & $\mathrm{pp}$ \\
\hline 5 & 1.04 & $35.6 \pm 0.2$ & 64 & $\mathrm{pp}$ \\
\hline 10 & 1.02 & $43.1 \pm 0.1$ & 60 & $\mathrm{pp}$ \\
\hline & & & & \\
\hline 1 & 1.05 & $29.7 \pm 0.0$ & 2924 & $\mathrm{cw}$ \\
\hline 5 & 1.05 & $34.1 \pm 0.1$ & 418 & $\mathrm{cw}$ \\
\hline 10 & 1.04 & $42.2 \pm 0.1$ & 127 & $\mathrm{cw}$ \\
\hline 15 & 1.01 & $46.9 \pm 0.1$ & 72 & $\mathrm{cw}$ \\
\hline
\end{tabular}

The oscilloscope trace of Figure 3 illustrates the way in which we implemented pulsed pumping. In this example, we used 50 - $\mu$ s-wide $\sim 2$-A current pulses at $5 \mathrm{kHz}$ repetition rate superimposed on a continuous drive current of $\sim 3 \mathrm{~A}$. (The particular driver that we used had an instanteous current limit of $5 \mathrm{~A}$ ). This corresponds to an instantaneous 938-nm pump power of $1.5 \mathrm{~W}$ pulsed and $2.3 \mathrm{~W}$ cw respectively. The oscilloscope trace shows the emission of the Q-switched pulse during the pump pulse interval. Depending on the exact resonator alignment (including etalon output coupler tilt) and precise cavity length, the Q-switched pulse emission occurred at different times within the pump pulse interval. Slightly increasing or decreasing the pump pulse width causes the Q-switched pulse emission to occur sooner or later in the pump window, consistent with the simulation results of Lai et al. ${ }^{26}$, and, as they point out, this could be used as a control parameter for a active feedback stabilization to compensate for long-term cavity length drift (due to temperature drift) to ensure that the microlaser reliably operates single mode for long periods of time. Our experience has been that with careful cavity alignment and active temperature stabilization of both the microlaser (to $\pm 0.01{ }^{\circ} \mathrm{C}$ ) and its pump diode (to $\pm 0.03{ }^{\circ} \mathrm{C}$ ), the microlaser can operate single frequency over several hours without any adjustments. If temperature or alignment drifts do cause unstable operation (i.e. shot-to-shot mode hopping) small adjustments to the pump pulse amplitude or width can be used to bring the microlaser back into reliable single-frequency operation.

A typical Q-switched output pulse waveform from our Yb:YAG microlaser is shown in Figure 4. The waveform was acquired using an Electro-Optics Technology ET-3500 InGaAs detector ( $>10 \mathrm{GHz}$ cutoff frequency) and a Tektronix TDS7404 digital oscilloscope (4 GHz front-end bandwidth and $20 \mathrm{GS} / \mathrm{s}$ sample rate). Although this waveform was recorded for $5-\mathrm{kHz}$ pulse-pumped operation, it is representative of all of the waveforms taken with this microlaser configuration. The pulse lengths varied from $\sim 1.05 \mathrm{~ns}$ (FWHM) for 1-kHz operation to $\sim 1.01 \mathrm{~ns}$ (FWHM) for $15-\mathrm{kHz}$ operation (see Table 1). We observed no after-pulses with the Yb:YAG microlaser that often plague Nd-based passively Q-switched microlasers.

The spatial beam quality produced by our Yb:YAG microlaser was excellent, as shown in the beam profile image presented in Figure 5. Although we did not directly measure $\mathrm{M}^{2}$ for this microlaser, we were consistently able to launch $>90 \%$ of the light into a single-mode fiber ${ }^{27}$ which we interpret as very good beam quality.

For many applications of microlasers, such as lidar and target ranging, pulse-to-pulse timing jitter is an important parameter. We used two methods to measure the timing jitter of our microlasers. First, we used a Stanford Research Systems model SR620 universal time counter which can measure the time interval between one pulse and the next pulse and compute the maximum, minimum, mean, and standard deviation for a sample of $\mathrm{N}$ pulse intervals. To corroborate 
the results provided by the SR620, we used the time interval measurement capability of our digital oscilloscope (in this case a TDS 5104B with 1-GHz front end and $5 \mathrm{GS} / \mathrm{s}$ digitizer rate) to produce histograms of time interval between a trigger pulse and the next pulse. In general, the results computed by the SR620 agreed well with those produced by the digital oscilloscope.

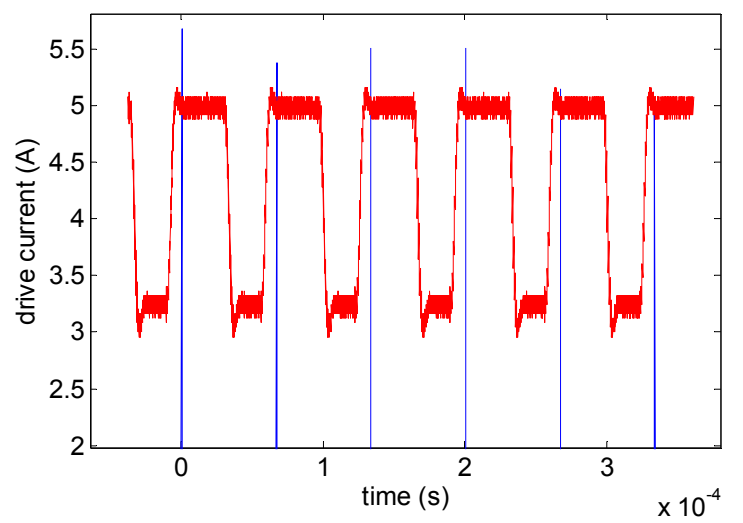

Figure 3 Example of pulsed pumping at $5 \mathrm{kHz}$ repetition rate. The red curve shows the drive current as a function of time. The blue sticks denote the Q-switched output pulses.

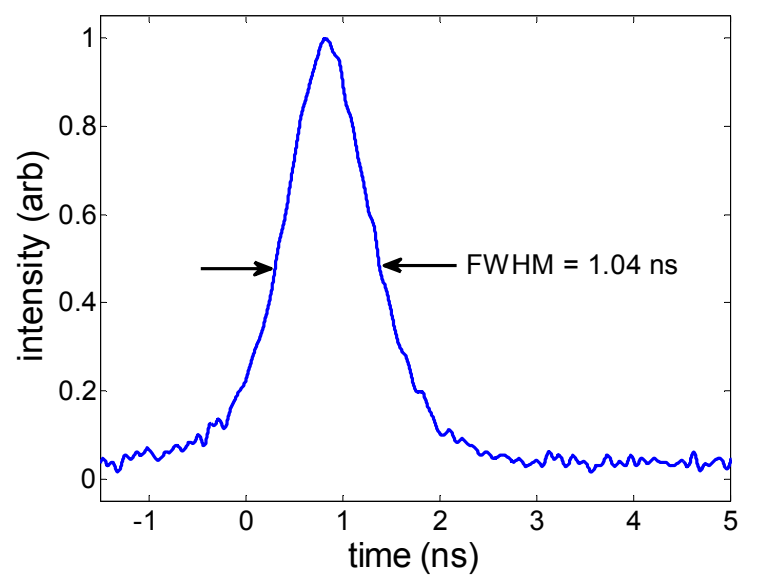

Figure 4 Temporal pulse shape for Yb:YAG microlaser operating pulse pumped at $5 \mathrm{kHz}$ repetition rate.

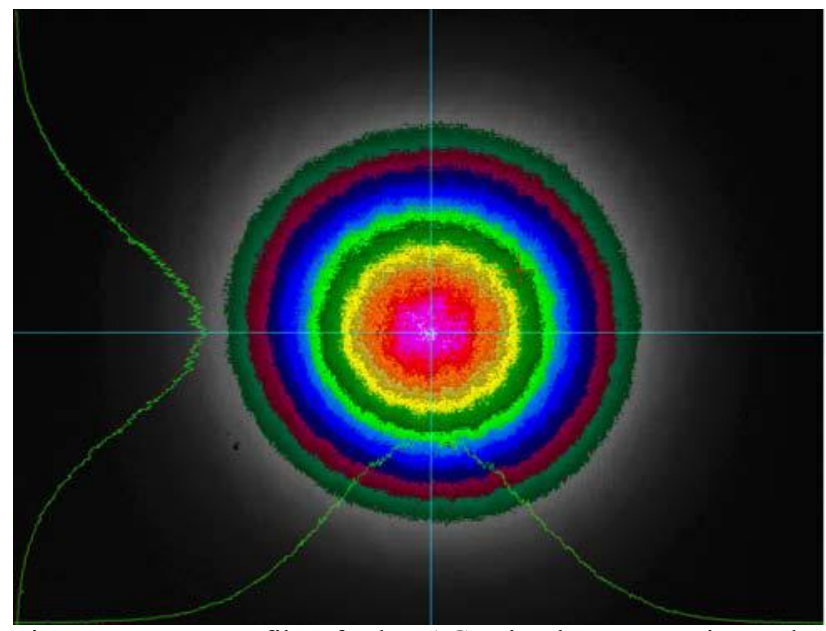

Figure 5 Beam profile of $\mathrm{Yb}: Y A G$ microlaser operating pulse pumped at $5 \mathrm{kHz}$ repetition rate.

In some respects the oscilloscope histograms provide more information than the SR620 since they show the shape of the pulse-to-pulse timing distribution. For example, Figure 6 shows a representative histogram produced by the oscilloscope when the microlaser was operating stably at $5 \mathrm{kHz}$ with pulsed pumping. This histogram, which represents $\sim 28 \mathrm{k}$ measurements taken over a 2-minute period, is symmetrical and shows a standard deviation of $63 \mathrm{~ns}$ (consistent with a measurement made with the SR620 over the same time period). In other cases, such as cw pumping (not shown in this paper), the distributions were not symmetrical because of a consistent repetition-rate drift over time.

We measured the pulse-to-pulse timing jitter of our microlasers for repetition rates from $1 \mathrm{kHz}$ to $15 \mathrm{kHz}$ for both pulsed pumping and $\mathrm{cw}$ pumping conditions. The results, plotted in Figure 7, show that pulsed pumping significantly reduces timing jitter, especially at lower repetition rates. We achieved $(1-\sigma)$ timing jitter as low as $60 \mathrm{~ns}$ RMS for $10-\mathrm{kHz}$ pulsepumped operation. Pulsed pumping was difficult to implement at repetition rates $>10 \mathrm{kHz}$ due to the frequency response 
of the laser diode driver. For cw pumping, the pulse-to-pulse timing jitter is a strong function of repetition rate with jitter as high as $\sim 3 \mu$ s for $1 \mathrm{kHz}$ operation reducing to near $60 \mathrm{~ns}$ for $15 \mathrm{kHz}$ operation. An additional benefit of pulsed pumping, not shown in the data here, is stabilization of the repetition rate ${ }^{26}$. For $\mathrm{cw}$ pumping, small, slow drift of the microlaser (or pump diode) temperature can cause corresponding drift in the repetition rate.

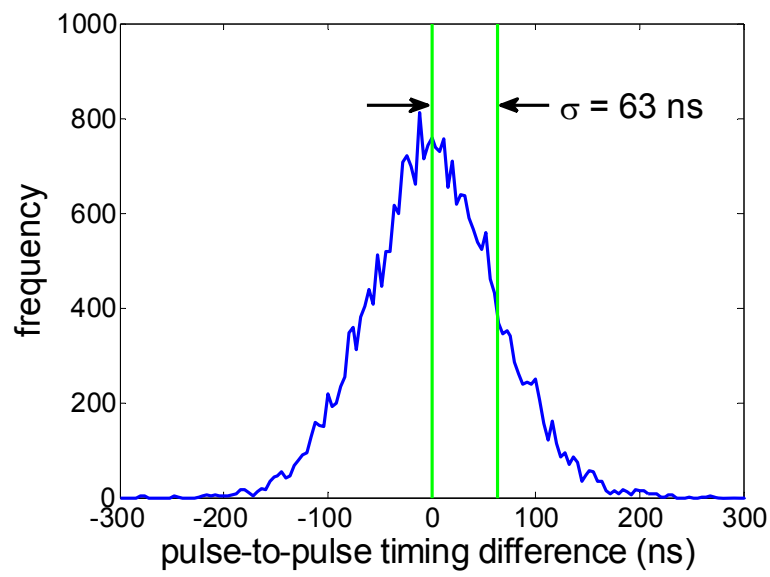

Figure 6 Pulse-to-pulse timing jitter histogram measured on Tektronix digital oscilloscope for $5 \mathrm{kHz}$ repetition rate and pulsed pumping. The histogram represents almost $28 \mathrm{k}$ points taken over a $\sim 2$-minute-long period.

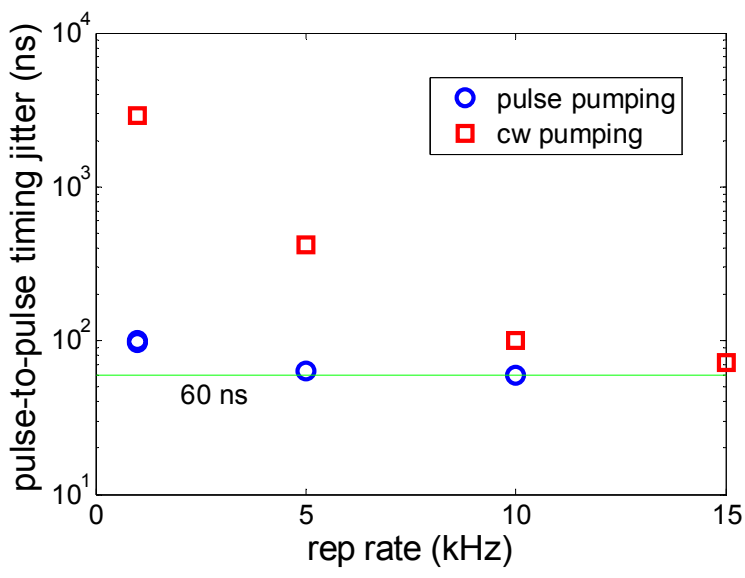

Figure 7 Pulse-to-pulse timing jitter vs. repetition rate for pulsed pumping and $\mathrm{cw}$ pumping. Note that at low repetition rates, pulsed pumping significantly reduces timing jitter.

It is worth reiterating that the pulse-to-pulse timing jitter of a passively Q-switched microlaser is drastically lower when it is reliably operating on a single longitudinal mode than when it is mode hopping shot to shot. As just discussed, pulsed pumping can further reduce shot-to-shot timing jitter. To our knowledge, we are the first to report that pulse-topulse timing jitter can be reduced even further by coiling the fiber delivering the pump light. We first noticed that stable operation of the Yb:YAG microlaser was strongly correlated with the precise position of the pump fiber and that movement or changes in bending of the pump fiber could cause significant changes in the microlaser output including causing stable single-mode operation to abruptly change to unstable shot-to-shot mode hopping. Even with careful temperature stabilization of the pump diode and microlaser and minimization of vibration and bending of the fiber, the pulse-to-pulse timing jitter was unstable over time. Also, the spatial beam profile was sensitive to the fiber bending. To understand the underlying causes of the instability, we imaged the output of the pump fiber onto a CCD and observed that the coarse speckle pattern changed when the fiber was bent or its position was changed. We also measured the farfield pattern from the pump fiber and found that its divergence was considerably less than the numerical aperture of the fiber. Clearly, the launch optics of the laser diodes do not fill all of the modes in the highly multi-mode delivery fiber, and any changes in the bending of the fiber cause re-distribution of the modes propagating in the fiber, resulting in changes in the spatial distribution of the pump light inside the gain medium. Also, the speckle pattern changed over short time periods even when the fiber was not moved, and we speculate that the spatial modes of the laser diode bar itself change over time, thereby exciting different modes of the delivery fiber at different times. By coiling the pump fiber (50 $\mathrm{mm}$ diameter in two orthogonal planes), we fill more propagating modes of the fiber, and, as a result, the pump power distribution at the end of the fiber (input to the microlaser) is improved spatially and is much less sensitive to subsequent motion of the fiber coils and changes in the laser diode bar output. Using the CCD, we observed that when the pump delivery fiber was coiled in two dimensions, the resulting speckle pattern was finer than when the fiber was straight, and that changes to fiber coil positions did not change the spatial distribution nearly as much as similar bending changes to the nominally straight fiber. By coiling the pump fiber we not only improved the microlaser's output beam quality and its pulse-to-pulse timing jitter, but also improved its short- and long-term operational stability by removing a potential source of change: unintentional changes of the pump irradiance distribution.

Figure 8 shows the Yb:YAG microlaser output energy vs. repetition rate for both $\mathrm{cw}$ and pulse pumped operation. Pulse pumped operation resulted in a small, but measurable increase in pulse energy. The pulse energy increased significantly as the repetition rate increased, probably due to the change in cavity parameters resulting from increased thermal lensing. The average power vs. repetition rate for these measurements is shown in Figure 9. The maximum average power of 
$\sim 700 \mathrm{~mW}$ was achieved at $15 \mathrm{kHz}$ repetition rate and corresponds to an optical-to-optical efficiency of $\sim 22 \%$ (or an electrical-to-optical efficiency of $\sim 8 \%$ ).

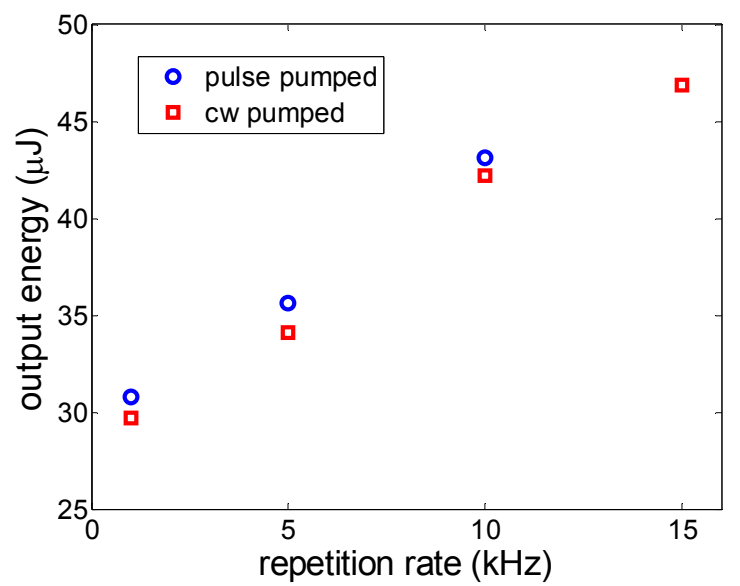

Figure 8 Output energy vs. repetition rate for both cw and pulse pumped operation of the $\mathrm{Yb}: \mathrm{YAG}$ microlaser.

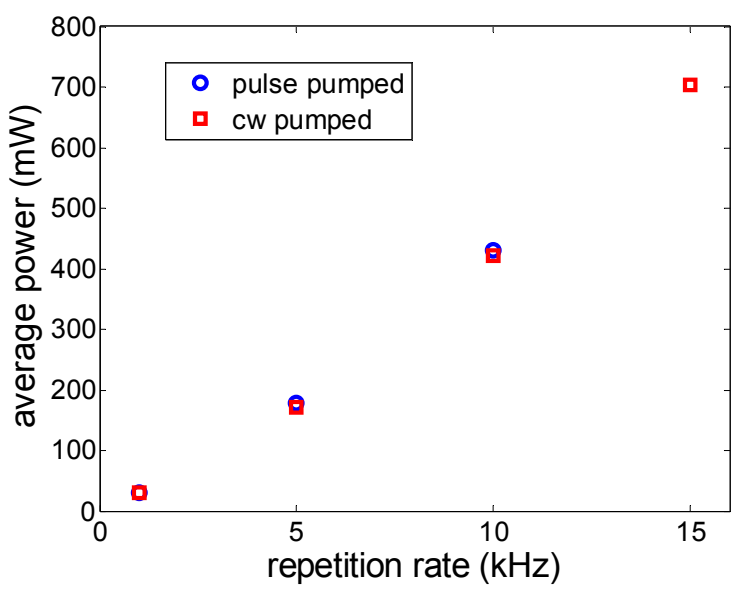

Figure 9 Average power vs. repetition rate for the Yb:YAG microlaser.

All of the data presented so far in this paper is from a single Yb:YAG microlaser configuration. We only changed the pump current (or pump current vs. time for pulsed pumping) to change the repetition rate. With this configuration, the maximum repetition rate at which we could reliably obtain single-longitudinal-mode operation was $15 \mathrm{kHz}$. At higher pump current (to produce higher repetition rate), the laser began mode hopping. We suspected that this repetition rate limit was due to changing thermal conditions inside the gain medium. In an effort to further increase the single-mode operating limit for this laser, we reconfigured the microlaser slightly. We replaced the aluminum heat sink with a copper heat sink for better heat extraction, we shortened the cavity length slightly, and we reduced the pump beam spot size inside the gain medium. In this configuration, we were able to achieve a maximum single-mode repetition rate of $19.4 \mathrm{kHz}$ with a pulse energy of $37 \mu \mathrm{J} / \mathrm{pulse}$ and a pulse width of $0.96 \mathrm{~ns}$ (FWHM). This corresponds to an average power of $718 \mathrm{~mW}$. The optical-to-optical conversion efficiency for this $19.4-\mathrm{kHz}$ configuration was $21 \%$ (approximately 7\% electrical-to-optical conversion efficiency). The optical pump power required to achieve $19.4-\mathrm{kHz}$ operation was only slightly greater than that required to obtain $15-\mathrm{kHz}$ operation with 1:1 pump imaging.

\section{SUMMARY}

We have designed, built, and characterized a diode-laser-pumped passively Q-switched Yb:YAG microlaser that is able to produce single-longitudinal-mode output near $1030 \mathrm{~nm}$ at repetition rates as high as $19.4 \mathrm{kHz}$. This microlaser uses a miniature intracavity Brewster polarizer to ensure linearly polarized output and it uses an undoped YAG etalon as its output coupler to permit single-mode operation at high repetition rates. We found that coiling the pump fiber improves the output beam quality, reduces the shot-to-shot timing jitter and greatly improves the short- and long-term stability of the microlaser output. This laser produced $\mathrm{TEM}_{00}$ spatial mode, 1-ns-long pulses with output energy of between $31 \mu \mathrm{J}$ and $47 \mu \mathrm{J}$ depending on repetition rate. Its pulse-to-pulse timing jitter was as low as $60 \mathrm{~ns}$ RMS for pulse pumped operation and nearly that low for cw-pumped operation at high repetition rate $(\geq 15 \mathrm{kHz})$. Its peak optical-to-optical efficiency is $22 \%$. By changing the saturable absorber Q-switch density, we have reconfigured this microlaser design to produce pulse lengths from $0.5 \mathrm{~ns}$ to $1.0 \mathrm{~ns}$. We have used this Yb:YAG microlaser to seed Yb-doped fiber amplifiers.

\section{ACKNOWLEDGEMENTS:}

We would like to thank Dahv Kliner, Roger Farrow, and Paul Schrader at Sandia National Laboratories in Livermore, CA for many useful discussions. We would also like to thank the skilled personnel at OptiSource, Inc. in Albuquerque, NM for their assembly of the miniature Brewster polarizers. This work was supported by Laboratory Directed Research 
and Development at Sandia National Laboratories. Sandia is a multiprogram laboratory operated by Sandia Corporation, a Lockheed Martin Company, for the United States Department of Energy's National Nuclear Security Administration under contract DE-AC04-94AL85000.

\section{REFERENCES:}

1. P. E. Schrader, J.-P. Fève, R. L. Farrow, D. A. V. Kliner, R. L. Schmitt, and B. T. Do, "Power scaling of fiberbased amplifiers seeded with microchip lasers," in Fiber Lasers V: Technology, Systems, and Applications, Proc. SPIE 6873, (2008).

2. S. Li, S. Zhou, P. Wang, Y. C. Chen, and K. K. Lee, "Self-Q-switched diode-end-pumped Cr,Nd:YAG laser with polarized output," Opt. Lett. 18, 203-205 (1993).

3. J. J. Zayhowski and C. Dill III, "Diode-pumped passively Q-switched picosecond microchip lasers," Opt. Lett. 19, 1427-1429 (1994).

4. J. J. Degnan, "Optimization of passively Q-switched lasers," IEEE J. Quantum Electron. 31, 1890-1901 (1995).

5. G. Xiao and M. Bass, "A generalized model for passively Q-switched lasers including excited state absorption in the saturable absorber," IEEE J. Quantum Electron. 33, 41-44 (1997).

6. X. Zhang, S. Zhao, Q. Wang, Q. Zhang, L. Sun, and S. Zhang, "Optimization of $\mathrm{Cr}^{4+}$-doped saturable-absorber Q-switched lasers," IEEE J. Quantum Electron. 33, 2286-2294 (1997).

7. F. D. Patel and R. J. Beach, "New formalism for the analysis of passively Q-switched laser systems," IEEE J. Quantum Electron. 37, 707-715 (2001).

8. T. Y. Fan, S. Klunk, and G. Henein, "Diode-pumped Q-switched Yb:YAG laser," Opt. Lett. 18, 423-425 (1993).

9. G. J. Spühler, R. Paschotta, M. P. Kullberg, M. Graf, M. Moser, E. Mix, G. Huber, C. Harder, and U. Keller, "A passively Q-switched Yb:YAG microchip laser," Appl. Phys. B 72, 285-287 (2001).

10. J. Dong, P. Deng, Y. Liu, Y. Zhang, J. Xu, W. Chen, and X. Xie, "Passively Q-switched Yb:YAG laser with $\mathrm{Cr}^{4+}: \mathrm{YAG}$ as the saturable absorber," Appl. Opt. 40, 4304-4307 (2001).

11. D. Y. Shen, D. Y. Tang, and J. Kong, "Passively Q-switched Yb:YAG laser with GaAs output coupler," Opt. Commun. 211, 271-275 (2002).

12. O. A. Buryy, S. B. Ubiszkii, S. S. Melnyk, and A. O. Matkovskii, "The Q-switched Nd:YAG and Yb:YAG microchip lasers optimization and comparative analysis,," Appl. Phys. B 78, 291-297 (2004).

13. E. P. Ostby, J. Fukumoto, R. D. Stultz, S. Matthews, and D. Filgas, "Short pulse and high repetition rate Qswitched Yb:YAG microchip laser," in Solid State Lasers XIV: Technology and Devices, H. J. Hoffman, and R. K. Shori, eds., Proc. SPIE 5707, 72-80 (2005).

14. J. Dong, A. Shirakawa, and K. Ueda, "Switchable pulses generation in passively Q-switched multilongitudinalmode microchip laser," Laser Phys. Lett. 4, 109-116 (2007).

15. J. Dong, A. Shirakawa, and K. I. Ueda, "Sub-nanosecond passively Q-switched Yb:YAG/ $/ \mathrm{Cr}^{4+}: \mathrm{YAG}$ sandwiched microchip laser," Appl. Phys. B 85, 513-518 (2006).

16. W. F. Krupke, "Ytterbium solid-state lasers - the first decade," IEEE J. Selected Topics in Quantum Electron. 6, 1287-1296 (2000).

17. F. D. Patel, E. C. Honea, J. Speth, S. A. Payne, R. Hutcheson, and R. Equall, "Laser demonstration of $\mathrm{Yb}_{3} \mathrm{Al}_{5} \mathrm{O}_{12}(\mathrm{YbAG})$ and materials properties of highly doped $\mathrm{Yb}: \mathrm{YAG}$, , IEEE J. Quantum. Electron. 37, 135144 (2001).

18. J. Dong, M. Bass, Y. Mao, P. Deng, and F. Gan, "Dependence of the $\mathrm{Yb}^{3+}$ emission cross section and lifetime on temperature and concentration in yttrium aluminum garnet," JOSA B 20, 1975-1979 (2003).

19. Y. F. Chen, and Y. P. Lan, "Comparison between c-cut and a-cut Nd:YVO4 lasers passively Q-switched with $\mathrm{Cr}^{4+}$ :YAG saturable absorber," Appl. Phys. B 74, 415-418 (2002).

20. E. P. Ostby, R. A. Ackerman, J. C. Huie, and R. L. Gentilman, "Ceramic Yb:YAG Microchip laser," in Solid State Lasers XV: Technology and Devices, H. J. Hoffman, and R. K. Shori, eds., Proc. SPIE 6100, 610004 (2006).

21. J. Dong, A. Shirakawa, K. Takaichi, K. Ueda, H. Yagi, T. Yanagitani, and A. A. Kaminskii, "All-ceramic passively Q-switched $\mathrm{Yb}: \mathrm{YAG} / \mathrm{Cr}^{4+}$ :YAG microchip laser," Electronics Letters 42, 1154-1155 (2006).

22. W. Koechner, Solid-State Laser Engineering (fifth edition, Springer-Verlag, 1999).

23. A. E. Siegman, Lasers (University Science Books, 1986). 
24. J. J. Zayhowski, C. Dill III, C. Cooke, and J. L. Daneu, "Mid- and high-power passively Q-switched microchip lasers," in OSA TOPS Advanced Solid State Lasers, M. M. Fejer, H. Injeyan, and U. Keller, eds. (1999).

25. J. B. Khurgin, F. Jin, G. Solyar, C. C. Wang, and S. Trivedi, "Cost-effective low timing jitter passively Qswitched diode-pumped solid-state laser with composite pumping pulses," Appl. Opt. 41, 1095-1097 (2002).

26. N. D. Lai, M. Brunel, F. Bretenaker, and A. LeFloch, "Stabilization of the repetition rate of passively Qswitched diode-pumped solid-state lasers," Appl. Phys. Lett. 79, 1073-1075 (2001).

27. P. E. Schrader, Sandia National Laboratories, Livermore, CA., personal communication (2007). 